\title{
Exosomal IncRNA SHNG7 Promotes High-Grade Serous Ovarian Cancer Progression and Function As ceRNA to Target Notch1 By Sponging miR-34a- $5 p$
}

Jing Cui

Shandong Qianfoshan Hospital

Xue Lu

Shandong University Qilu Hospital

Weiting Gu

Shandong University Qilu Hospital

Cunzhong Yuan

Shandong University Qilu Hospital

Hui Zhang ( $\nabla$ williamzh@126.com )

Shandong University Qilu Hospital

\section{Research}

Keywords: high-grade serous ovarian cancer, IncRNA SHNG7, exosome, Notch 1, miRNA-34a-5p

Posted Date: November 9th, 2021

DOI: https://doi.org/10.21203/rs.3.rs-1031640/v1

License: (c) (i) This work is licensed under a Creative Commons Attribution 4.0 International License.

Read Full License 


\section{Abstract}

Serous ovarian cancer, especially high-grade serous ovarian cancer (HGSOC) has a high mortality rate, and its five-year survival rate is only $30 \%$. The reason for the difficulty in diagnosis and treatment is that the origin and pathogenic mechanism of HGSOC are still poorly understood. In this study, we tried to explore the molecular mechanism of IncRNA SHNG7 in regulating proliferation, invasion and migration in HGSOC. The expression of IncRNA SHNG7 was upregulated in cancer tissues, and was closely correlated with poor outcomes. LncRNA SHNG7 promoted cell proliferation, invasion, migration and influenced the cell cycle of cancer cell lines. Furthermore, IncRNA SHNG7 could function as competitive endogenous RNA (ceRNA) via directly sponging of microRNA-34a-5p, which further regulating the expression of Notch 1. Moreover, IncRNA SHNG7 could be carried by exosomes and the exosomal IncRNA SHNG7 promoted angiopoiesis. Taken together, our results proved that IncRNA SHNG7 could function as ceRNA and contribute to HGSOC progression, which provided a novel prognostic and therapeutic marker for HGSOC.

\section{Introduction}

Ovarian cancer is a common malignant tumor in women, with the fifth highest mortality rate. Serous ovarian cancer, especially high-grade serous ovarian cancer (HGSOC) has a high mortality rate (90\%), and its five-year survival rate is only $30 \%$ (1). One of the main reasons for the high mortality rate of ovarian cancer is that most patients are in the advanced stage when they are discovered, and metastasis is existed (2). The reason for the difficulty in diagnosis and treatment is that the origin and pathogenic mechanism of HGSOC are still poorly understood. In this study, we tried to explore the molecular mechanism in HGSOC.

Long non-coding RNAs (IncRNAs) are a class of transcripts with more than 200 nucleotides in length and no protein-coding capacity. Emerging evidences proved that IncRNAs act as key regulators of target gene expression in various biological processes, such as gene transcription, RNA splicing, and RNA transport and translation (3). And aberrant expression of IncRNAs has been found involved in tumor occurrence, progression and metastasis (4). It is proved that some IncRNAs could function as competing endogenous RNAs (ceRNAs) via sponging microRNAs (miRNAs)to regulate the expression of specific genes (5). In HGSOC, several IncRNAs have been identified as key roles in the initiation or progression. For example, upregulation of IncRNA SOCAR promoted proliferation, migration and invasion in ovarian cancer cells (6). LncRNA NEAT1 was proved to promote cell proliferation and migration by sponging miR-506 in HGSOC (7). While in this study, the function of IncRNA SHNG7 was studied in HGSOC.

Exosomes are a type of membranous vesicles with a diameter of about $30 \sim 100 \mathrm{~nm}$, which are released from cells into the extracellular matrix. Many kinds of cells could release exosomes (8). Exosomes could function as a carrier for multiple messenger RNAs, microRNA, IncRNA and circRNA. Exosomes could be isolated from serum, urine, bile and breast milk $(9,10)$. More and more studies have shown that the exosomes of tumor cells are related to the occurrence and deterioration of tumors (11). They can regulate 
immune function, promote tumor angiogenesis, invasion and metastasis, and even directly affect other tumors or non-tumor cells, thereby affecting the fate of the cell or tissue (12).

In the present study, we found IncRNA SHNG7 was dyregulated in HGSOC tissues, and its overexpression dramatically promoted cell proliferation, migration and invasion. LncRNA SHNG7 could be released from cells via exosomes, and exosomal IncRNA SHNG7 was demonstrated promoting angiogenesis. Besides, we identified that IncRNA SHNG7 could function as ceRNA to target Notch1 by competitively sponging miR-34a-5p. These findings provide new insights into the molecular functions of IncRNA SHNG7 and shed new light on the treatment of HGSOC.

\section{Materials And Methods}

\section{Tumor tissues}

A total of $30 \mathrm{HGSOC}$ tissues and 20 normal ovarian epithelium tissues were collected from the First Affiliated Hospital of Shandong First Medical University. None of patients was treated with chemotherapy or radiotherapy before surgery. Samples were washed with sterile phosphate buffered saline and then frozen in liquid nitrogen. Tumor stage was determined according to the FIGO staging system. This study was approved by the Ethic Committee of the First Affiliated Hospital of Shandong First Medical University.

Cell culture and transfection

Human EOC cell lines A2780 and SKOV3 were obtained from American Type Culture Collection (ATCC) and maintained using Dulbecco's modified Eagle's medium (Gibco, Carlsbad, CA, USA) supplemented with $10 \%$ fetal bovine serum (FBS). All cells were incubated in a humidified atmosphere containing $5 \% \mathrm{CO}_{2}$ at $37^{\circ} \mathrm{C}$. Vectors that stably overexpressed IncRNA SHNG7, siRNA targeting IncRNA and SHNG7, miR-34a$5 p$ mimics, miR-34a-5p inhibitor and controls were transfected into cells using Lipofectamine 2000 reagent (Invitrogen, Carlsbad, CA) according to the manufacturer's instructions.

Quantitative RT-PCR analysis

Total RNA was extracted from tissues and cells using Trizol reagent (Invitrogen) according to the manufacturer's protocol. Extracted RNA was reversely transcribed into cDNA using a PrimeScriptTM RT Reagent Kit (TaKaRa Bio, China) following the manufacturer's instructions. qRT-PCR was carried out using a SYBR Premix Ex Taq ${ }^{\text {TM }}$ Kit (Takara). U6 or GAPDH was used as an internal control. The relative expression was determined using the $2^{-\Delta \Delta C T}$ method.

Cell proliferation

Cell proliferation was detected using Cell Counting Kit-8 (CCK-8; Dojindo, Kumamoto, Japan) according to the manufacturer's protocol. Cells were seeded into 96-well plates. After incubation for the indicated time, 
$20 \mu \mathrm{L}$ CCK-8 solution was added into each well and incubated for $2 \mathrm{~h}$. Absorbance values at $490 \mathrm{~nm}$ were measured on a Microplate Reader (Bio-Rad).

Cell cycle assay

Cell cycle analysis was performed using flow cytometry. Cells were collected and wash twice with PBS $(1 \times)$, and fixed with $70 \%$ ethanol at $-20^{\circ} \mathrm{C}$ for $24 \mathrm{~h}$. Cells were incubated with RNase $A$ at $37^{\circ} \mathrm{C}$ for $30 \mathrm{~min}$, and then stained with $400 \mu \mathrm{L}$ propidium iodide on ice for $30 \mathrm{~min}$. Cell cycle distribution was analyzed using a BD FACSCalibur ${ }^{\text {TM }}$ flow cytometer (BD Biosciences).

Transwell assay

Cell migration and invasion were completed with a Transwell system (Corning, NY, USA). A total of $10^{4}$ cells in $200 \mu \mathrm{L}$ of serumfree medium were added to the upper chamber. For invasion assay, Matrigel was additional coated on the upper chamber. The lower chamber was filled with $600 \mu \mathrm{l}$ medium with $20 \%$ FBS. After incubation, cells on the upper chamber were removed and the cells on the lower surface were fixed with $20 \%$ methanol and stained with $0.1 \%$ crystal violet. The cells were observed under a microscope.

Western blot

Cells were collected and lysed in RIPA buffer containing protease inhibitors. Proteins were separated by $8 \%$ SDS-PAGE then transferred onto PVDF membranes (Millipore, USA). Then the membranes were blocked with $5 \%$ nonfat milk for $1 \mathrm{~h}$ at room temperature, and incubated with primary antibodies overnight at $4^{\circ} \mathrm{C}$. Subsequently, the membranes were incubated with secondary antibodies for $1.5 \mathrm{~h}$. the protein bands were detected using the Pro-lighting horseradish peroxidase (HRP) agent. The expression of $\beta$-actin was used as the internal control.

\section{Luciferase Assay}

The dual-luciferase miRNA target expression vector pmirGLO (Promega, Madison, Wisconsin) was used to generate luciferase reporter constructs. Wild-type (SHNG7-WT and Notch1-WT) and mutant-type (SHNG7-MT and Notch1-MT) vectors were constructed. Cells were cotransfected with miR-34a-5p mimics (or NC) and wild-type (or mutant-type) vectors using Lipofectamine 2000. Luciferase activity was examined using a luciferase reporter assay kit (Transgen Biotech, Beijing, China). Each group was performed in triplicate.

Isolation of exosomes

Exosomes were isolated from cell culture medium by differential centrifugation. Cells and other debris were removed by centrifugation at $300 \mathrm{~g}$ and $3,000 \mathrm{~g}$. Shedding vesicles were removed from supernatant by centrifuged at 10,000 g. Finally, supernatant was centrifuged at 110,000g and exosomes were 
obtained. Isolation of exosomes from serum was performed using ExoQuick Plasma prep and Exosome precipitation kit (SBI, USA).

Tumor formation assay in nude mice

NCG (NOD-Prkdcem26Cd52 IL2 ${ }^{\text {rgem26Cd22 } / G p t) ~ m i c e ~ w e r e ~ p u r c h a s e d ~ f r o m ~ N B R I ~ o f ~ N a n j i n g ~ U n i v e r s i t y ~}$ (Nanjing, China), which were maintained in a pathogen-free facility. Cells overexpressing SHNG7 were trypsin digested, washed with PBS, and then resuspended in PBS. Then 200 $\mu$ l of the suspended cells $\left(1 \times 10^{7}\right)$ were injected into the armpit or peritoneal cavity of each mouse.3-4 weeks later, the mice were sacrificed and tumor weight or metastasis number were examined. All the animal experiments were performed with the approval of the Shandong First Medical University Animal Care and Use Committee.

Statistical Analyses

Each experiment was repeated in triplicate independently. Values were shown as mean $\pm S D$. Statistical analysis was performed using GraphPad Prism 6 (GraphPad Software, USA). $P$ values $<0.05$ were considered statistically significant.

\section{Results}

LncRNA SHNG7 is upregulated in HGSOC and correlates with poor outcomes

The expression of IncRNA SHNG7 was firstly detected in HGSOC tissues. Compared with normal ovarian epithelium tissues $(n=20)$, IncRNA SHNG7 was significantly upregulated in HGSOC tissues $(n=30)$ as detected by qRT-PCR (Figure 1A). To analyze the effect of IncRNA SHNG7 on the prognosis of HGSOC, the Kaplan-Meier Plotter was applied. The HGSOC patients were divided into two groups, high and low group, based on the median expression value of IncRNA SHNG7. As shown in Figure 1B, the survival rate in high level group was consistently lower than that in low level group.

LncRNA SHNG7 promotes cell proliferation

Cell proliferation ability was detected by CCK-8 assay. A2780 cells were transfected with siRNA of IncRNA SHNG7 (or NC) and SKOV3 cells were transfected with IncRNA SHNG7 overexpression vectors (or NC). The successful transfection was confirmed by qRT-PCR (Figure 1C). In A2780 cells, the inhibition of IncRNA SHNG7 significantly suppressed cell proliferation compared with NC group, and in SKOV3 cells, the overexpression of IncRNA SHNG7 significantly promoted cell proliferation compared with NC group (Figure 1D).

LncRNA SHNG7 promotes cell migration and invasion

To detect the effect of IncRNA SHNG7 on cell migration and invasion, Transwell assay was applied. The Transwell assay results showed that overexpression of IncRNA SHNG7 dramatically promoted cell migration and invasion in SKOV3 cells (Figure 2A). The transfection of siRNA targeting IncRNA SHNG7 
dramatically inhibited cell migration and invasion in A2780 cells (Figure 2B). Then cell cycle distribution of A2780 cells and SKOV3 cells was analyzed using flow cytometry. As shown in Figure 2C, overexpression of IncRNA SNHG7 reduced the proportion of cells in G1 phase and inhibition of IncRNA SNHG5 increased the proportion the G1 phase. In addition, we detected the expression of epithelialmesenchymal transition (EMT)-associated proteins and cell cycle related protein markers (CDK4 and CDK6). The western blot assay showed that overexpression of IncRNA SHNG7 could upregulate the ETM markers such as $\mathrm{N}$-cadherin, $\beta$-Catenin, Vimentin, CDK4 and CDK6, and downregulate the epithelial marker E-cadherin (Figure 2D).

LncRNA SHNG7 could function as ceRNA via directly sponging miR-34a-5p

The subcellular location of IncRNA SHNG7 was detected by nuclear mass separation experiment, and we found that IncRNA SHNG7 was mainly located in cytoplasm in A2780 cells (Figure 3A). According to starBase website, miR-34a-5p was estimated as a potential target of IncRNA SHNG7 (Figure 3B). To confirm whether IncRNA SHNG7 directly interacted with miR-34a-5p, luciferase reporter assay was performed. As shown in Figure 3C, miR-34a-5p significantly reduced the luciferase activity in cells transfected with SHNG7 WT reporter, but not in cells with SHNG7 MT reporter.

LncRNA SHNG7 is carried by exosomes and promotes angiogenesis

Exosomes were isolated from serum and observed by transmission electron microscopy (Figure 4A). The exosomes was verified based on the specific marker protein (CD63 and TSG101) (Figure 4B). Then we isolated exosomes from medium of SKOV3 cells transfected with IncRNA SHNG7 overexpression vectors or control vectors. Compared with exosomes from SKOV3 cells (NC), the relative expression of IncRNA SHNG7 in exosomes from SKOV3 cells (SHNG7) was significantly increased (Figure 4C). These results indicated that exosomes are potential carriers of IncRNA SHNG7 in HGSOC. Then we cultured HUVECs with medium from SKOV3 cells (SHNG7) or SKOV3 cells (NC) to detect the role of exosomal IncRNA SHNG7 on angiogenesis in vitro. The ring formation assay showed that HUVECs transfected with exosomal IncRNA SHNG7 had a clear tendency toward ring formation compared with control group (Figure 4D). And the IncRNA SHNG7 upregulated angiogenesis related protein markers, such as Notch 1, VEGF A and DII4 (Figure 4E).

miR-34a-5p directly targets Notch1

Based on the predictive results of TargetScan 7.1, Notch 1 was a putative target gene of miR-34a-5p. One potential miR-34a-5p binding site was found in the 3'UTR of Notch 1 (Figure 5A). Luciferase reporter was applied to verify the prediction. The luciferase reporter assay showed that miR-34a-5p significantly declined the luciferase activity of Notch 1-WT reporter, but no considerable change was observed in Notch 1-MT reporter (Figure 5B). Immunohistochemical staining was performed to compare the expression level of Notch 1 in the HGSOC tissues $(n=30)$ and normal control samples $(n=20)$. It was found that Notch 1 had higher intensity of immunostaining in HGSOC (76.7\%) than in normal control (20.0\%) (Figure 5C and D). 
LncRNA SHNG7 enhances the growth and metastasis in vivo

Compared with the NC group, the average weight of tumor formed by cells overexpressing SHNG7 was much higher, which indicated that SHNG7 enhanced the growth of ovarian cancer cells in vivo (Figure 6A and B). Consistently, SHNG7 significantly increased the number of peritoneal metastasis in NCG mice (Figure 6C and D).

\section{Discussion}

Recent studies have demonstrated that IncRNAs play an important role in various physiological and pathological processes. In many diseases, especially cancer, IncRNAs are often released from control (13). In the past few years, a large number of IncRNAs have been discovered in mammalian transcriptomes (14). Emerging evidences proved that IncRNA SHNG7 functions as oncogene in human cancers and positively related to clinicopathological characteristics and poor prognosis of patients (15). LncRNAs that are enriched in the cytoplasm could regulate gene expression at the post-transcriptional level via interacting with miRNAs (16). In several cancers, IncRNA SHNG7 was found in the cytoplasm, such as glioblastoma, colorectal cancer (17) and prostate cancer (18). Similarly in this study, we identified that IncRNA SHNG7 was located in cytoplasm in A2780 cells. And IncRNA SHNG7 was demonstrated function as a ceRNA to sponge miR-34a-5p and regulate its target gene Notch 1. In HGSOC tissues, IncRNA SHNG7 was upregulated. And the overexpression of IncRNA SHNG7 dramatically promoted cell proliferation, migration and invasion. These evidences indicated that IncRNA SHNG7 act as an oncogene in HGSOC.

Notch signaling pathway is a highly conserved signaling pathway that determines the fate of cells (19). Its receptors and ligands are type I transmembrane proteins that could regulate cell functions through cell-cell interactions (20). In mammals, the Notch signaling pathway includes four receptors (Notch1Notch4). Notch 1 is an important member of the Notch family (21). Recent studies showed that Notch 1 is not only important for normal cell differentiation (22), its pathophysiological changes are also related to the occurrence and development of some tumors (23). In most cases, activation of Notch signal has oncogenic effects in vitro and animal modules (Capobianco, 1997 \#181). In a variety of cancers, Notch 1 is found dysregulated (Nefedova, 2004 \#182). Studies have shown that Notch plays an important role in the development of follicles and corpus luteum (Vorontchikhina, 2005 \#183). The expression of activated Notch1 and its downstream hes1 gene in ovarian adenocarcinoma is significantly higher than that in ovarian adenoma and normal ovarian tissue (Hopfer, 2005 \#180), indicating that the Notch signaling pathway (especially Notch 1 ) is closely related to the development of ovarian cancer.

Between tumor cells or other cells in the body, such as lymphocytes or antigen recognition cells, exosomes rely on the special transmitter components contained in them to transmit special biological signals, thereby affecting the proliferation, migration and invasion ability of tumor cells (Yanez-Mo, 2015 \#189). More and more studies have shown that the exosomes in the microenvironment of tumor cells are related to the occurrence and development of tumor cells (Lowry, 2015 \#185). These exosomes can 
promote the proliferation, invasion and metastasis of tumor cells by regulating the body's immune response and promoting neovascularization in tumors, in addition, exosomes secreted by these tumor cells can directly act on other tumor or non-tumor cells (Boelens, 2014 \#184). In the present study, we demonstrated the presence of IncRNA SHNG7 in exosomes from serum of HGSOC patients and medium of cancer cells. And the exosomal IncRNA SHNG7 could promote angiopoiesis of HUVECs.

In conclusion, we identified the carcinogenic role of IncRNA SHNG7 in HGSOC. The overexpression of IncRNA SHNG7 significantly promoted cell proliferation, migration and invasion. LncRNA SHNG7 could be carried to serum or medium from cancer cells, and the exosomal IncRNA SHNG7 dramatically promoted angiopoiesis. Besides, we identified that IncRNA SHNG7 could function as a ceRNA against miR-34a-5p, which further regulating Notch 1. The newly identified IncRNA SHNG7/ miR-34a-5p / Notch 1 axis provides novel insight into the proliferation and metastasis of HGSOC and represents a potential therapeutic target for the clinical treatment of HGSOC.

\section{Declarations}

\section{Ethics approval and consent to participate}

This study was approved by the Ethics Committee of the First Affiliated Hospital of Shandong First Medical University. All participants were recruited after prociding a signed informed consent.

\section{Consent for publication}

Not available.

\section{Availability of data and materials}

The data used and/or analysis during the current study are available from the corresponding author on reasonable request.

\section{Competing interests:}

The authors declare that they have no competing interests.

\section{Funding}

This study was supported by the National Natural Science Foundation of China (81301789), the Shandong Province Natural Science Foundation (ZR2011HQ013هZR2020MH273), the Key Research and Development Projects in Shandong Province, China (2016GSF201150).

\section{Author's contributions}

This study was conceived, designed and interpreted by ZH. CJ and LX undertook the data acquisition, analysis, and interpretation. YCZ and GWT were responsible for the comprehensive technical support. CJ 
contributed to the inspection of data and final manuscript. All authors read and approved the final manuscript.

\section{Acknowledgements}

We sincerely thank all participants in the study.

\section{References}

1. Lengyel E. Ovarian cancer development and metastasis. The American journal of pathology 2010; 177: 1053-1064.

2. Hennessy B T, Coleman R L and Markman M. Ovarian cancer. Lancet 2009; 374: 1371-1382.

3. Tang Y, Cheung B B, Atmadibrata B, et al. The regulatory role of long noncoding RNAs in cancer. Cancer letters 2017; 391: 12-19.

4. Prensner J R, lyer M K, Sahu A, et al. The long noncoding RNA SChLAP1 promotes aggressive prostate cancer and antagonizes the SWI/SNF complex. Nature genetics 2013; 45: 1392-1398.

5. Kong $X$, Duan $Y$, Sang $Y$, et al. LncRNA-CDC6 promotes breast cancer progression and function as ceRNA to target CDC6 by sponging microRNA-215. Journal of cellular physiology $2019 ; 234$ : $9105-$ 9117.

6. Guo Q, Wang L, Zhu L, et al. The clinical significance and biological function of IncRNA SOCAR in serous ovarian carcinoma. Gene 2019; 713: 143969.

7. Yong W, Yu D, Jun Z, et al. Long noncoding RNA NEAT1, regulated by LIN28B, promotes cell proliferation and migration through sponging miR-506 in high-grade serous ovarian cancer. Cell death \& disease 2018; 9: 861.

8. Andre Mdo R, Pedro A and Lyden D. Cancer Exosomes as Mediators of Drug Resistance. Methods in molecular biology 2016; 1395: 229-239.

9. Vader P, Breakefield X $O$ and Wood M J. Extracellular vesicles: emerging targets for cancer therapy. Trends in molecular medicine 2014; 20: 385-393.

10. Skog J, Wurdinger T, Van Rijn S, et al. Glioblastoma microvesicles transport RNA and proteins that promote tumour growth and provide diagnostic biomarkers. Nature cell biology 2008; 10: 14701476.

11. Lowry M C, Gallagher W M and O'driscoll L. The Role of Exosomes in Breast Cancer. Clinical chemistry 2015; 61: 1457-1465.

12. Boelens M C, Wu T J, Nabet B Y, et al. Exosome transfer from stromal to breast cancer cells regulates therapy resistance pathways. Cell 2014; 159: 499-513.

13. Rui Q, Xu Z, Yang P and He Z. Long noncoding RNA expression patterns in lymph node metastasis in colorectal cancer by microarray. Biomedicine \& pharmacotherapy = Biomedecine \& pharmacotherapie 2015; 75: 12-18. 
14. Bu D, Yu K, Sun S, et al. NONCODE v3.0: integrative annotation of long noncoding RNAs. Nucleic acids research 2012; 40: D210-215.

15. Zhou Y, Tian B, Tang J, et al. SNHG7: A novel vital oncogenic IncRNA in human cancers. Biomedicine \& pharmacotherapy $=$ Biomedecine \& pharmacotherapie 2020; 124: 109921.

16. Schmitt A M and Chang H Y. Long Noncoding RNAs in Cancer Pathways. Cancer cell 2016; 29: 452463.

17. Shan Y, Ma J, Pan Y, Hu J, Liu B and Jia L. LncRNA SNHG7 sponges miR-216b to promote proliferation and liver metastasis of colorectal cancer through upregulating GALNT1. Cell death \& disease 2018; 9: 722.

18. Qi H, Wen B, Wu Q, et al. Long noncoding RNA SNHG7 accelerates prostate cancer proliferation and cycle progression through cyclin D1 by sponging miR-503. Biomedicine \& pharmacotherapy $=$ Biomedecine \& pharmacotherapie 2018; 102: 326-332.

19. Artavanis-Tsakonas S, Rand M D and Lake R J. Notch signaling: cell fate control and signal integration in development. Science 1999; 284: 770-776.

20. Nickoloff B J, Osborne B A and Miele L. Notch signaling as a therapeutic target in cancer: a new approach to the development of cell fate modifying agents. Oncogene 2003; 22: 6598-6608.

21. Saravanamuthu S S, Gao CY and Zelenka P S. Notch signaling is required for lateral induction of Jagged1 during FGF-induced lens fiber differentiation. Developmental biology 2009; 332: 166-176.

22. Cao L, Arany P R, Wang Y S and Mooney D J. Promoting angiogenesis via manipulation of VEGF responsiveness with notch signaling. Biomaterials 2009; 30: 4085-4093.

23. Lefort $K$, Mandinova A, Ostano P, et al. Notch1 is a p53 target gene involved in human keratinocyte tumor suppression through negative regulation of ROCK1/2 and MRCKalpha kinases. Genes \& development 2007; 21: 562-577.

\section{Figures}


A

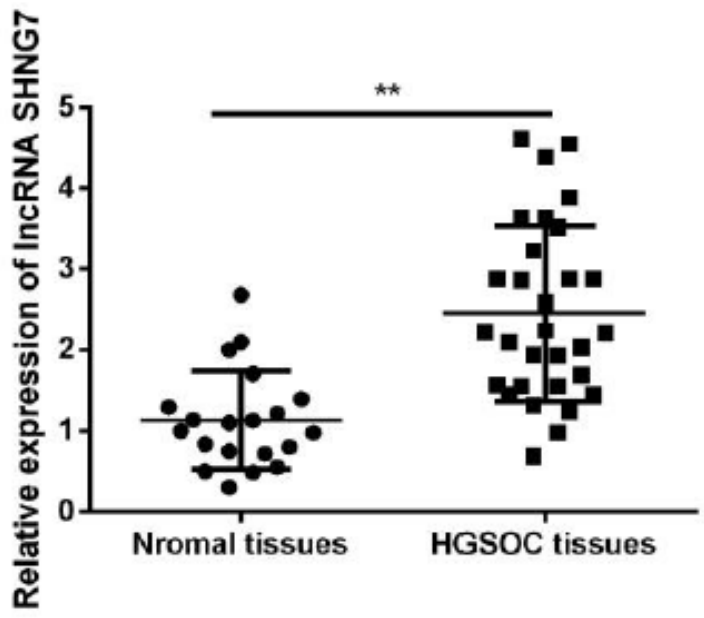

B

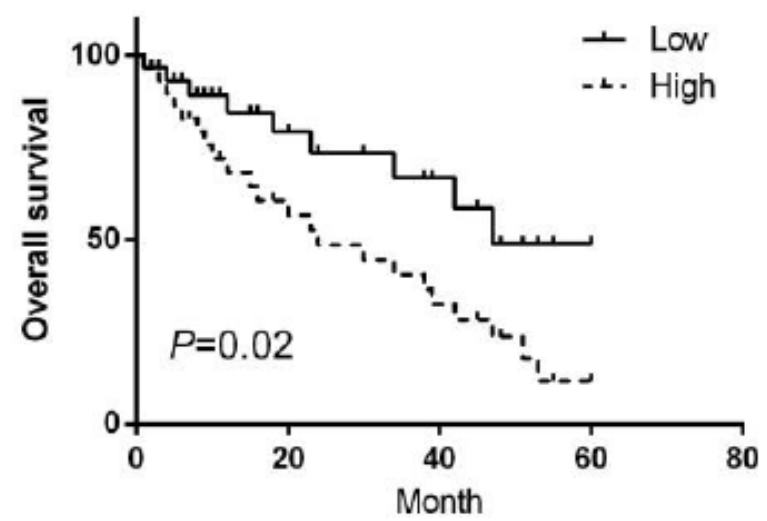

C

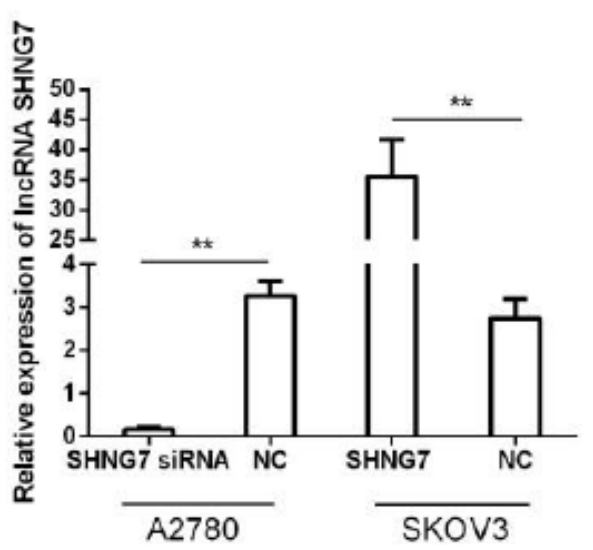

D
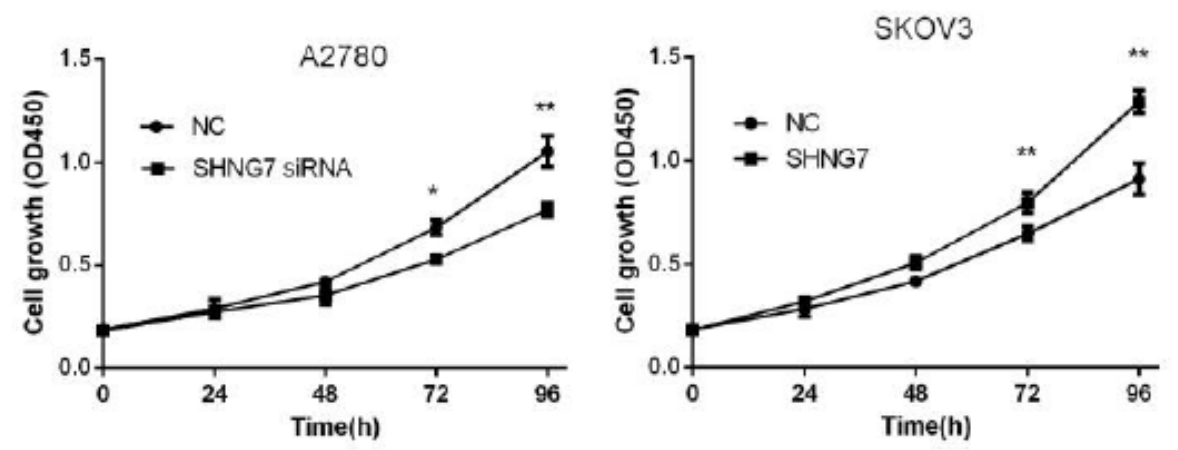

Figure 1

LncRNA SNHG7 was upregulated in high-grade serous ovarian cancer (HGSOC). A. The IncRNA SNHG7 expression was elevated in HGSOC tissues. B. The relationship between IncRNA SNHG7 and the survival of HGSOC patients. C. The expression of IncRNA SNHG7 in A2780 cells (NC and siRNA) and SKOV3 cells (NC and overexpression vector). D. LncRNA SNHG7 promoted cell proliferation. 
A

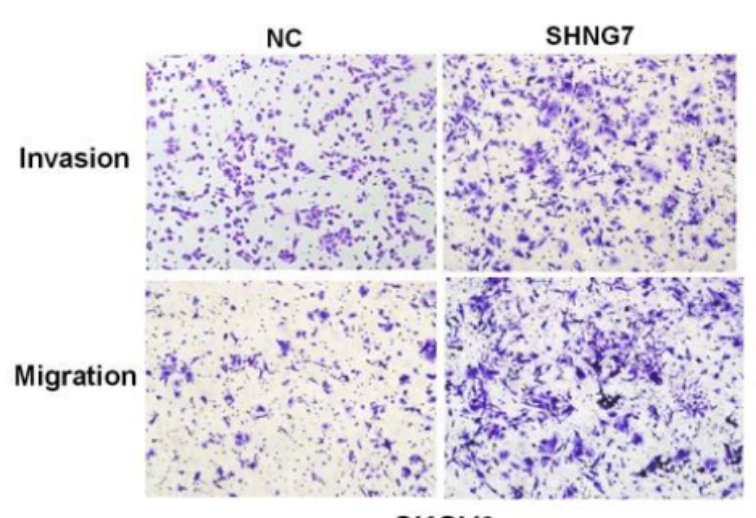

SKOV3

C
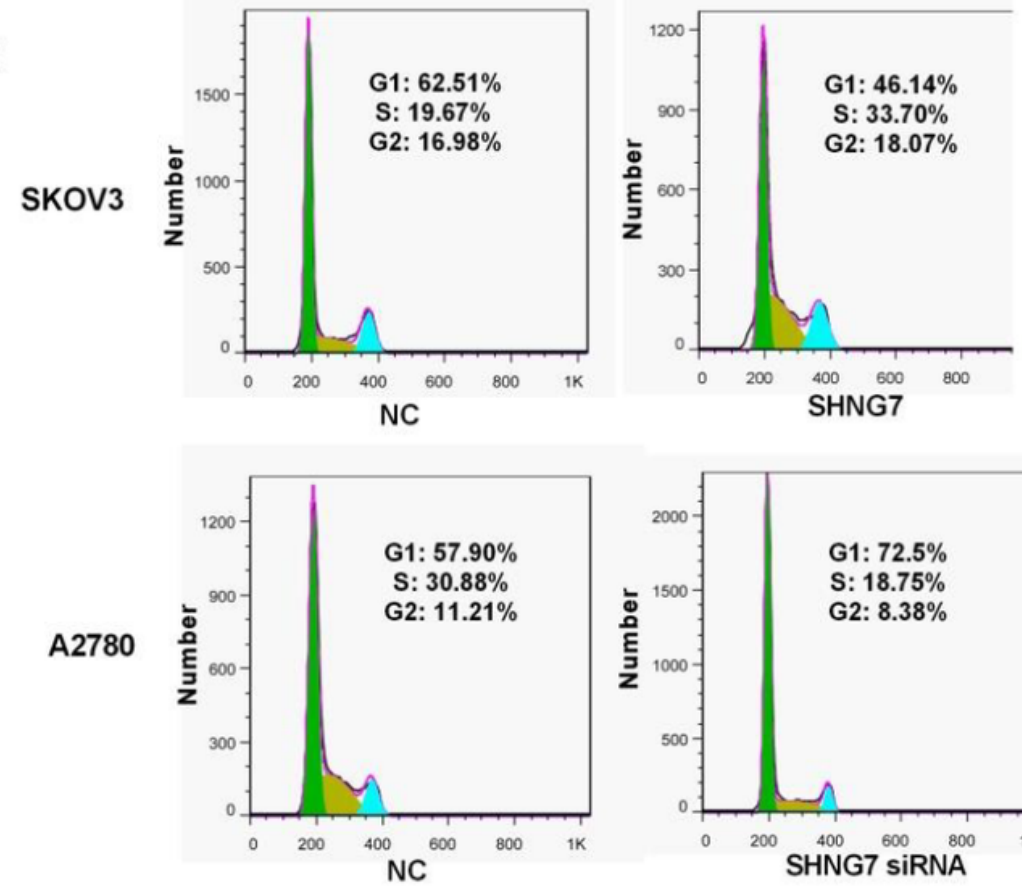

B

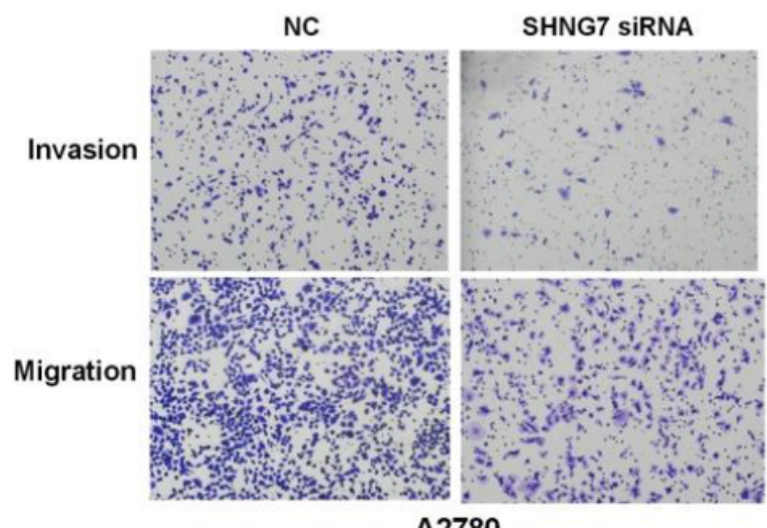

A2780

D

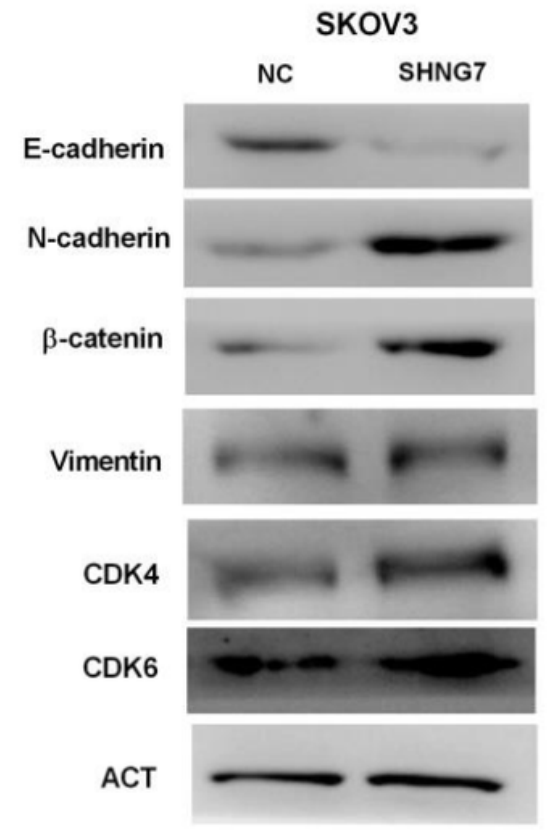

\section{Figure 2}

LncRNA SNHG5 promoted cell migration and invasion. A. Effect of IncRNA SNHG5 on migration and invasion in SKOV3 cells. B. Effect of IncRNA SNHG5 siRNA on migration and invasion in A2780 cells. C. Flow cytometry verified the cell cycle of A2780 cells and SKOV3 cells. D. Western blot. 
A

A2780

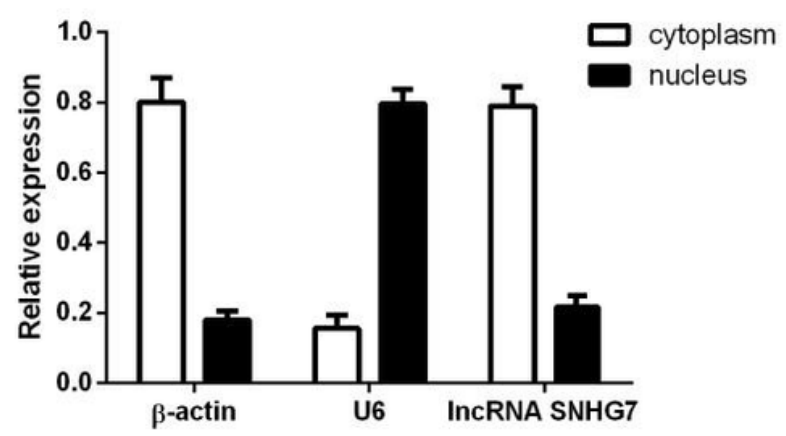

C

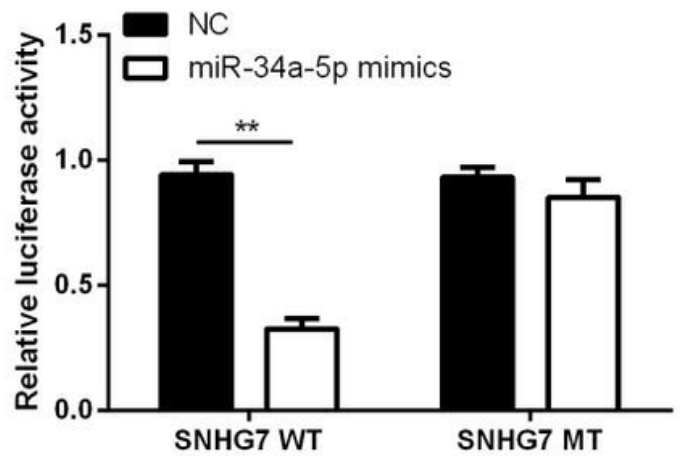

B

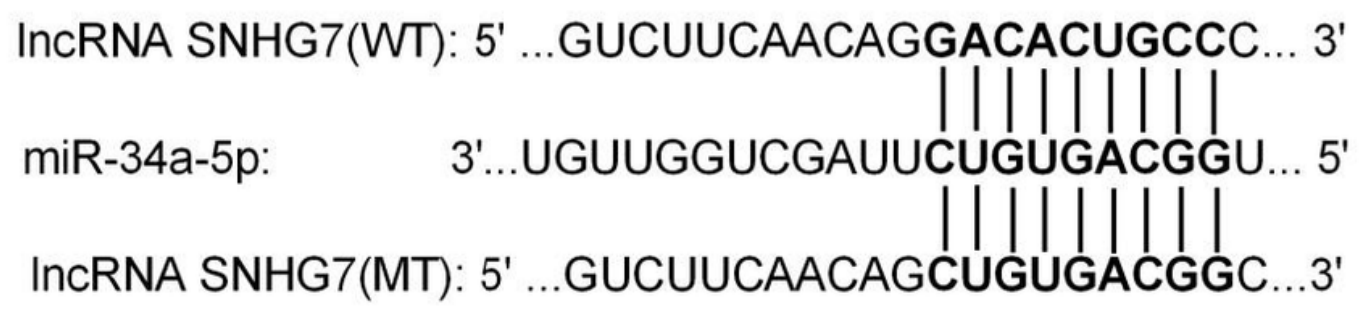

Figure 3

LncRNA SNHG7 could function as a competing ceRNA for miR-34a-5p. A. LncRNA SNHG7 was mainly located in cytoplasm in A2780 cells. B. The predicted targeting sequence of miR-34a-5p on IncRNA SNHG7. C. Luciferase reporter assay in A2780 cells. 
A

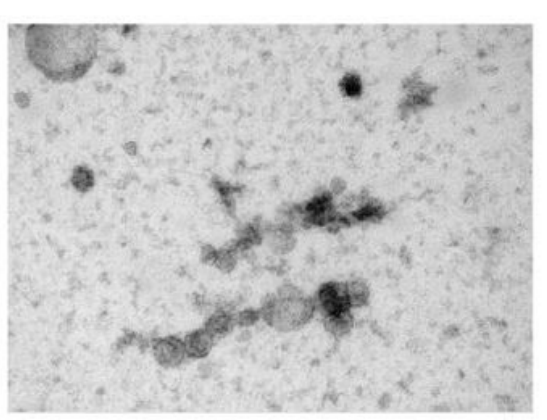

B

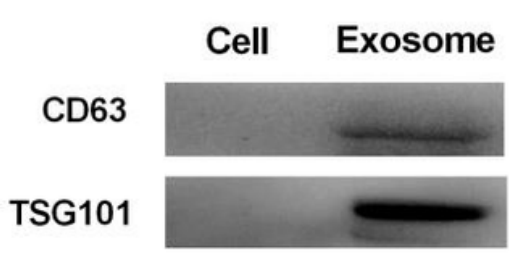

C

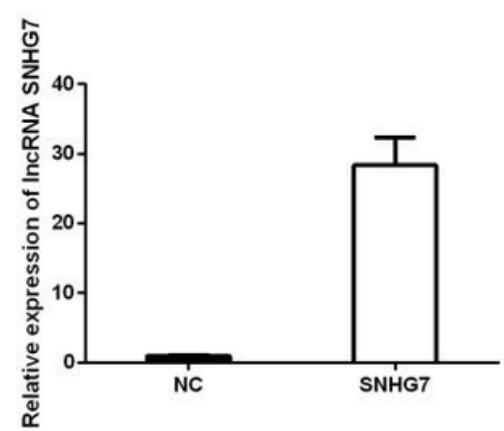

E

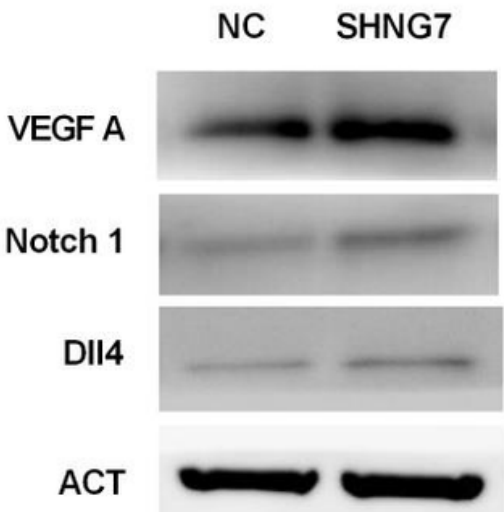

Figure 4

Exosomal IncRNA SNHG7 promoted angiogenesis. A. Exosomes viewed by electron microscopy. B. Specific protein markers of exosomes were detected by western blot. C. Relative expression of IncRNA SNHG7 in exosomes derived from SKOV3 cells (NC and SNHG7 vector). D. Ring formation of HUVECs. E. Western blot. 
A

Notch 1 (WT): 5' ...UUUUACACAGAAACACUGCC... miR-34a-5p: 3'...UGUUGGUCGAUUCUGUGACGGU Notch 1 (MT): 5'...UUUUACACAGAAAGUGACGG...

\section{C}

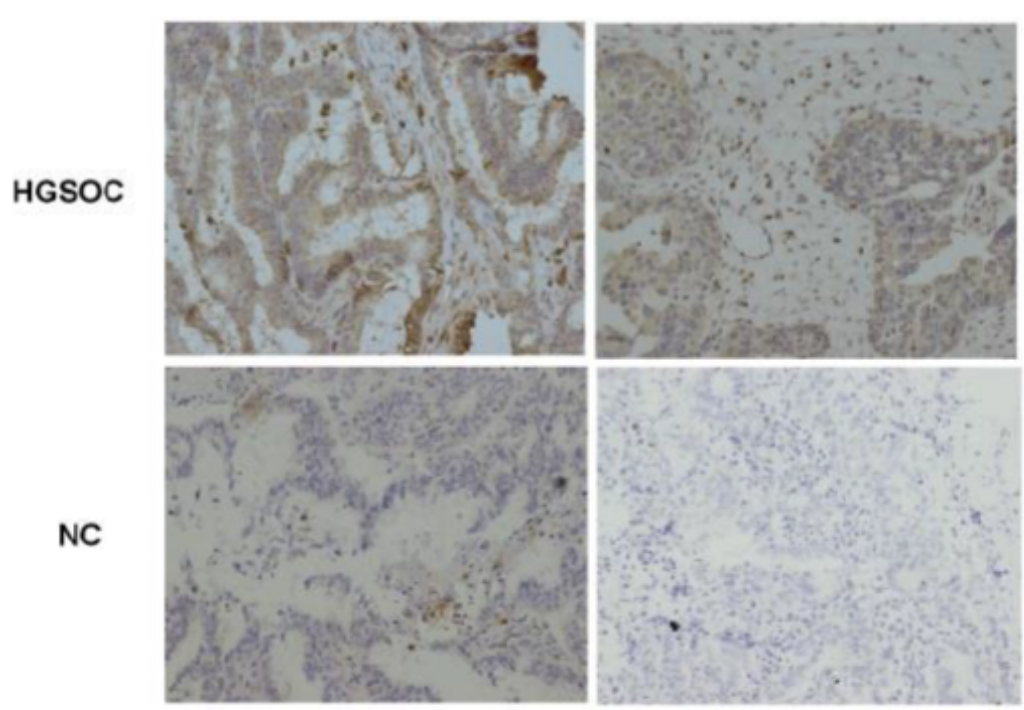

B

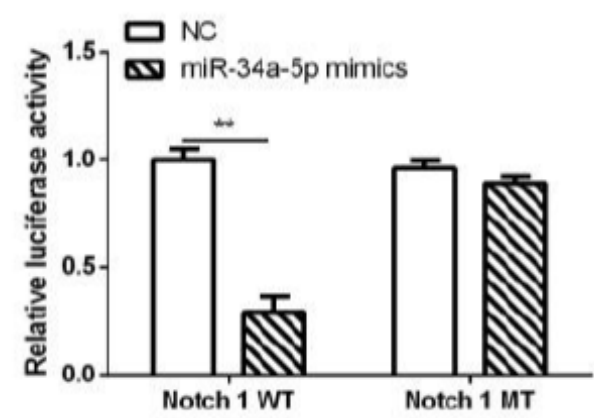

D

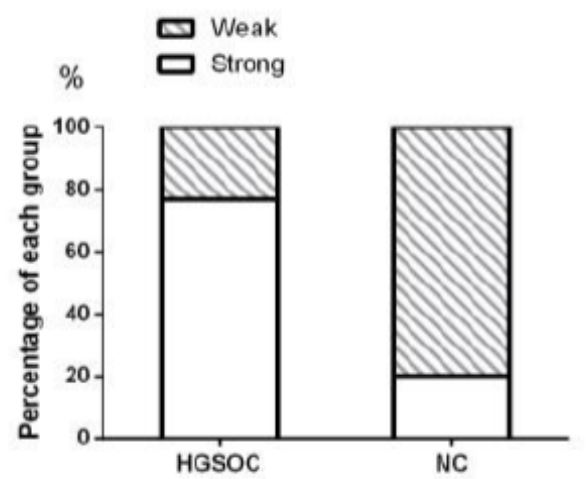

\section{Figure 5}

Notch 1 was identified as target of miR-34a-5p. A. The predicted targeting sequence of miR-34a-5p on Notch 1. B. Luciferase reporter assay with wild type (WT) or mutant type (MT) of Notch 1. C.

Representative images of immunohistochemical staining of Notch 1 in the cancer tissues and normal tissues ( $\times 100$ magnification). D. The distribution of immunohistochemical score, indicates that Notch 1 has a significantly higher expression in HGSOC. 
A

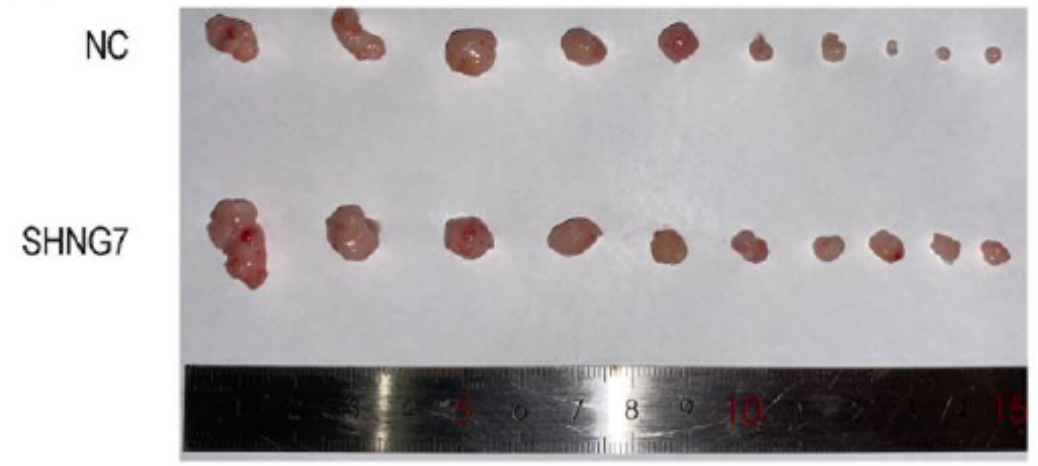

C

NC

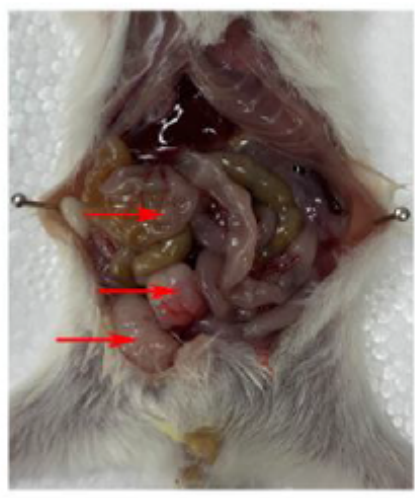

B

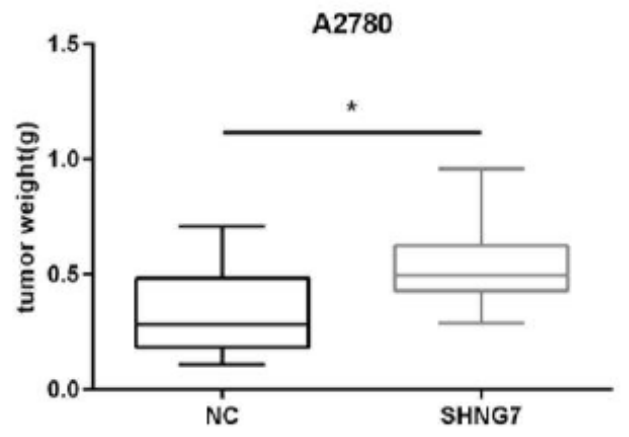

D

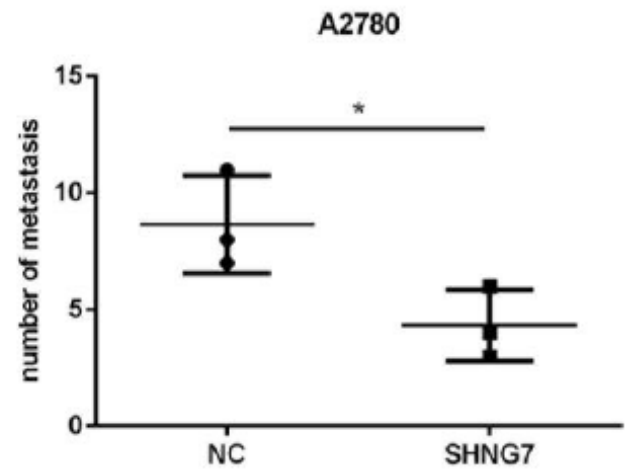

\section{Figure 6}

LncRNA SNHG5 enhanced tumor growth and metastasis in vivo. A. The photograph of tumors harvested from different groups. B. The tumor weights were compared between two groups (data are mean \pm SEM, $\left.{ }^{*} p<0.05, n=10\right)$. C. Abdominal metastasis nodes (red arrow) of two group mice. D. SNHG5 increased the number of metastasis (data are mean $\pm S E M,{ }^{*} p<0.05, n=3$ ). 